\title{
Health-Adjusted Life Expectancy among Canadian Adults with and without Hypertension
}

\author{
Lidia Loukine, ${ }^{1}$ Chris Waters, ${ }^{1}$ Bernard C. K. Choi, ${ }^{1,2,3}$ and Joellyn Ellison ${ }^{1}$ \\ ${ }^{1}$ Centre for Chronic Disease Prevention and Control, Public Health Agency of Canada (PHAC), Government of Canada, \\ Ottawa, ON, Canada K1A 0 K9 \\ ${ }^{2}$ Department of Epidemiology and Community Medicine, University of Ottawa, Ottawa, ON, Canada \\ ${ }^{3}$ Shantou University Medical College, Shantou 515041, China \\ Correspondence should be addressed to Bernard C. K. Choi, bernard.choi@phac-aspc.gc.ca
}

Received 19 October 2010; Accepted 11 April 2011

Academic Editor: Christina Chrysohoou

Copyright () 2011 Lidia Loukine et al. This is an open access article distributed under the Creative Commons Attribution License, which permits unrestricted use, distribution, and reproduction in any medium, provided the original work is properly cited.

Hypertension can lead to cardiovascular diseases and other chronic conditions. While the impact of hypertension on premature death and life expectancy has been published, the impact on health-adjusted life expectancy has not, and constitutes the research objective of this study. Health-adjusted life expectancy (HALE) is the number of expected years of life equivalent to years lived in full health. Data were obtained from the Canadian Chronic Disease Surveillance System (mortality data 2004-2006) and the Canadian Community Health Survey (Health Utilities Index data 2000-2005) for people with and without hypertension. Life table analysis was applied to calculate life expectancy and health-adjusted life expectancy and their confidence intervals. Our results show that for Canadians 20 years of age, without hypertension, life expectancy is 65.4 years and 61.0 years, for females and males, respectively. HALE is 55.0 years and 52.8 years for the two sexes at age 20; and 24.7 years and 22.9 years at age 55 . For Canadians with hypertension, HALE is only 48.9 years and 47.1 years for the two sexes at age 20; and 22.7 years and 20.2 years at age 55 . Hypertension is associated with a significant loss in health-adjusted life expectancy compared to life expectancy.

\section{Background}

Hypertension or high blood pressure (HTN) is an important risk factor for cardiovascular disease. In 2002, the World Health Organization estimated that at least $50 \%$ of cases with cardiovascular disease and $75 \%$ of strokes were caused by elevated blood pressure [1]. Hypertension is considered to be one of the leading risk factors for death and disability worldwide.

The number of Canadians with hypertension is increasing in Canada. The first report on hypertension from the Canadian Chronic Disease Surveillance System published by Public Health Agency of Canada shows that the agestandardized prevalence of hypertension among the adult population increased by about 7\% between 1998 and 2006 and is projected to increase by another $25 \%$ by the end of 2012 [2]. According to that report the crude prevalence of diagnosed hypertension among Canadian adults (20 years old and older) in 2006 was $22.7 \%$. Similar estimates of the prevalence of hypertension are also reported by Statistics Canada based on results from the first cycle of Canadian Health Measure Survey [3]. In many cases, the presence of hypertension leads to premature death and therefore the reduction of life expectancy (LE) and health expectancy (healthy life expectancy). Because of high morbidity in the hypertensive population, the reporting of mortality or life expectancy which uses only mortality experience is not enough to fully describe the burden of the chronic condition in population.

This paper reports results on Health-Adjusted Life Expectancy (HALE). HALE is an indicator that can be used as a summary measure of population health. While LE is the average number of years a person is expected to live, HALE is life expectancy weighted or adjusted for the level of Health Related Quality of Life (HRQOL). It combines morbidity and mortality data in one single indicator of population health and indicates the average time that a person could expect to live in a healthy state. Comparison of disparities 
in LE and HALE for a population of people with and without hypertension, and evaluation of loss in LE and the proportion of life lived in poor health for these cohorts can provide a comprehensive picture of how hypertension changes the lives of people.

There are few published studies which looked at the impact of hypertension on life expectancy $[4,5]$ and, to our knowledge, no previously published study has looked at high blood pressure and health expectancy. This is the first population-based Canadian study of life expectancy and healthadjusted life expectancy for people with and without diagnosed hypertension.

\section{Data Sources and Methods}

Health-adjusted life expectancy is a composite indicator that combines morbidity and mortality into a single statistic. Mortality data files from the Canadian Chronic Disease Surveillance System (CCDSS) were used for estimating age and sex-specific mortality rates. While mortality data and population counts are sufficient for calculating life expectancy, a measure of health-related quality of life is also needed to estimate health-adjusted life expectancy, its variance and corresponding $95 \%$ confidence intervals. The measure used in this analysis was the Health Utilities Index Mark 3 which was estimated from the Canadian Community Health Survey (CCHS).

2.1. Canadian Chronic Disease Surveillance System. The Canadian Chronic Disease Surveillance System is a collaborative network of provincial and territorial chronic disease surveillance systems, supported by the Public Health Agency of Canada [6]. It was created to improve the breadth of information about the burden of chronic diseases in Canada so that policy makers, researchers, health practitioners, and the general public could make better public and personal health decisions. CCDSS collects data from various sources on the prevalence, incidence, mortality, and utilization of health care services related to hypertension and other chronic conditions and diseases.

In each province and territory, the health insurance registry database is linked to the physician billing and hospitalization databases to generate summarized data for residents of Canada who have used the Canadian health care system. These summarized data are stored in the CCDSS for routine analysis. If there is sufficient evidence of use due to hypertension, it is assumed that a person had been diagnosed with hypertension. The minimum requirement was at least 1 hospitalization or 2 physician claims over a 2 -year period with specific code(s) for hypertension from the International Classification of Diseases (ICD) (ICD-9 codes 401-405; ICD10 codes I10- I13 and I15). With respect to hypertension, the CCDSS collects data for the adult population only, that is, 20 years old and older.

For Canadian adults with and without hypertension, agespecific mortality rates for all causes are included in the CCDSS. For this study, the age-specific mortality rates for persons with and without adult hypertension were used to calculate life expectancy and health-adjusted life expectancy.
The study was limited to persons who are 20 years old and older.

2.2. Canadian Community Health Survey. The Canadian Community Health Survey is an ongoing cross-sectional national health survey, conducted by Statistics Canada [79], that collects information related to health status, health care utilization, and health determinants for the Canadian population. It includes a large sample of about 130,000 respondents and is designed to provide reliable estimates at the local health region level.

Prior to 2007, data collection occurred every 2 years for a 12-month period. After major changes to the survey design in 2007, data collection now occurs on an ongoing (monthly) basis with annual releases. Data are available for 2000/2001, 2003, 2005, 2007, 2008, and 2009.

The CCHS produces an annual microdata file and a file combining 2 years of data. The survey collection years can also be combined by users to examine subpopulations of rare characteristics. The respondents of the first three cycles of the survey were asked if they have any of 26-30 chronic conditions including hypertension. The survey data include information for persons aged 12 years and older. The survey does not include people who live in institutions or in remote areas. The household-level response rate in 2005 was $84.9 \%$, and the person-level response rate was 92.5\% [9].

As a measure of health-related quality of life, the Health Utilities Index Mark 3 from the following three CCHS data files was used for this study: (1) cycle $1.12000 / 2001$ share file [7]; (2) cycle 2.12003 subsample 1 file [8]; and (3) cycle 3.1 2005 subsample 1 file [9].

2.3. Health Utilities Index Mark 3. HUI3 is a multiattribute utility measure that defines health states according to eight attributes (vision, hearing, speech, ambulation, dexterity, emotion, cognition, and pain), with five or six levels ranging from normal to severely limited functioning for each. Single attribute utility scores range from 0.0 (lowest level of functioning) to 1.0 (full functional capacity) [10]. The eight attributes are combined into a single score using the multiattribute utility function:

$u=1.371 *\left(b_{1} * b_{2} * b_{3} * b_{4} * b_{5} * b_{6} * b_{7} * b_{8}\right)-0.371$,

where $u$ is HUI 3 and $b_{i}$ is a single attribute utility score.

The overall scores on the HUI 3 range from -0.36 (the worst possible HUI3 health state), through 0.0 (death), to 1.0 (perfect health). From a societal perspective, some health states are considered worse than dead, and consequently are assigned negative scores. Differences of 0.03 or more in overall HUI3 scores and 0.05 or more in single-attribute utility scores are considered to be clinically important [11].

Sex and age-specific HUI averages were estimated from a combined CCHS data file for persons with and without hypertension. The bootstrap methodology [12], recommended by Statistics Canada, was used to calculate the variance estimate. Both the point estimates and variance estimations were calculated using the BOOTVARE_V31 macro [13]. 
2.4. Survey Sample Sizes. All three cycles of the CCHS (cycle 1.1 share file, cycle 2.1 subsample, and cycle 3.1 subsample) were combined by the pooled method to increase the sample size and to decrease variation in the estimates [14]. The sample size for the combined file, which included people 20 years and older and spanned the years 2000 through 2005, was 173,567 (32,612 with hypertension; 140,955 without hypertension).

Mortality data for Quebec and Nunavut were unavailable from the CCDSS, and health utilities index data from the Northwest Territories and Nunavut were unavailable from the 2000/2001 CCHS (cycle 1.1) survey file. Therefore, these jurisdictions were excluded from all analyses.

2.5. Life Table Analysis. The Chiang method [15] was used to generate period (2004-2006) life tables by diseasespecific/disease-deleted populations and by sex using the 14 standard age groups $(20-24,25-9, \ldots, 80-85,85+$ years $)$. The Gompertz function was used to provide an accurate estimate of life expectancy for the last open-ended 85+ age interval, in order to close the life table. This method was described by Hsieh [16]. The modified Sullivan method [17] was applied for the HALE calculation. According to this method the "life years lived" was adjusted by the HUI

$$
L_{x}^{\prime}=L_{x} * \mathrm{HUI}_{x},
$$

where $L_{x}^{\prime}$ is adjusted life years lived in age interval $x, L_{x}$ is life years lived in age interval $x$, and $\mathrm{HUI}_{x}$ is Health Utilities index for people in age interval $x$.

The variance of LE was calculated by the Chiang method. The variance in HALE, for the population without hypertension, was calculated by the Bebbington method [18] taking into account just the variability of HUI. The sample size of the population without hypertension was large; therefore the variance of the life-table itself was close to zero and did not contribute much to the variance of HALE. However, the sample size of population with hypertension was relatively small and the life-table variance was quite noticeable and could not be ignored. Therefore the variance of HALE for the population with hypertension was calculated taking into account both the variability of the life table and the variability of HUI. This method was introduced by Mathers [19]. All calculations were performed using specially developed SAS macros. The 95\% confidence intervals for LE and HALE were built based on the normality assumption. $Z$ tests were used to test the statistical significance of loss in life expectancy and the absolute difference in life expectancy. The absolute difference in life expectancy is defined as the difference between life expectancy and the health adjusted life expectancy (LE-HALE) for the same cohort.

\section{Results}

Figures 1 and 2 show age and sex-specific estimates of the total (LE) and the healthy (HALE) number of years of life remaining for adults without and with hypertension. As expected, higher values for both LE and HALE were obtained
TABLE 1: Difference in life expectancy (LE) and health-adjusted life expectancy (HALE) between females and males with and without hypertension (HTN) for selected ages, Canada, 2004 to 2006. (Dataset for this study excluded Quebec, Nunavut and Northwest Territories.)

\begin{tabular}{lcc}
\hline & LE $(\mathrm{F})-\mathrm{LE}(\mathrm{M})$ & HALE(F)-HALE $(\mathrm{M})$ \\
\hline \multicolumn{3}{c}{ At age of 20 years } \\
\hline With HTN & $5.2^{*}$ & $1.8^{* *}$ \\
Without HTN & $4.4^{*}$ & $2.2^{*}$ \\
\hline \multicolumn{3}{c}{ At age of 55 years } \\
\hline With HTN & $4.2^{*}$ & $2.5^{*}$ \\
Without HTN & $3.6^{*}$ & $1.8^{*}$ \\
\hline \multicolumn{3}{c}{ At age of 85 years } \\
\hline With HTN & 2.0 & 0.9 \\
Without HTN & 1.7 & 0.8 \\
\hline
\end{tabular}

${ }^{*}$ Statistically significant $(P$-value $<.0001)$.

$* *$ Statistically significant $(P$-value $<.05)$.

for people not diagnosed and who did not report hypertension. For 20-year-old females who were not diagnosed with hypertension, LE was estimated to be an additional 65.4 years and HALE was an additional 55 years (Figure 1). The corresponding LE and HALE for males were 61.0 and 52.8 years, respectively. Within the population of people with hypertension, the LE and HALE were, respectively, 62.1 and 48.9 years for females and 56.9 and 47.1 years for males (Figure 2).

Figure 3 illustrates differences in age- and sex-specific average Health Utilities Index for persons with and without hypertension. HUI reported by men was higher than that reported by women for both populations. The index decreased with age and was lower for persons with disease.

The difference in LE between female and male populations with hypertension varies between 2.0 and 5.2 years across age groups, higher than that in the population without hypertension (Table 1). Differences in HALE between sexes were approximately half the difference in LE. HALE differences between females and males with hypertension were between 0.9 and 2.5 years. Similar patterns were observed for persons without hypertension. The narrower difference in HALE was a result of men reporting better health (higher HUI score) than women. Many LE and HALE differences reported in Table 1 were statistically significant.

Table 2 illustrates absolute and relative measures of unhealthy years of life as well as the loss in LE and HALE associated with hypertension within age-sex grouping. The age groups selected for presentation were, the beginning of life table (age 20 years), the average age of hypertension incidence ( 55 years), and the last age interval of the life table (85 years). As age increases, the number of unhealthy years of life decreases while the proportion of unhealthy life years remaining increases. The unhealthy years of life for persons with hypertension varied between 13.2 years (at age 20) and 3.6 years (at age 85+) for females and between 9.8 years (at age 20) and 2.5 years (at age 85+) for males which was much higher than that for persons without hypertension. The corresponding number of unhealthy years for person 


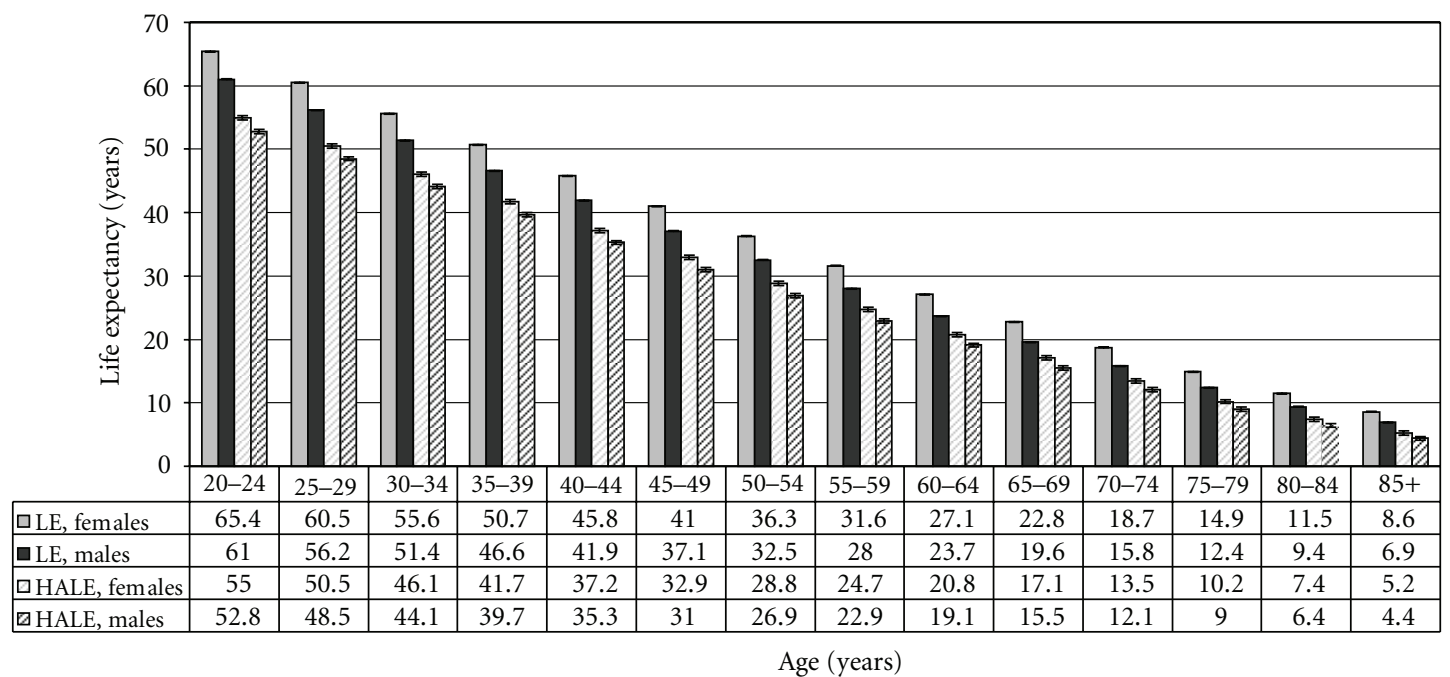

Figure 1: Life expectancy (LE) and health adjusted life expectancy (HALE) for females and males, without hypertension, Canada, 2004 to 2006. (Data source: Canadian Community Health Survey Data Files (CCHS) from Statistics Canada, 2000-2005 and Canadian Chronic Disease Surveillance System Data Files (CCDSS) from Public Health Agency of Canada, 2004-2006. Data Files excluded Quebec, Nunavut and Northwest Territories.)

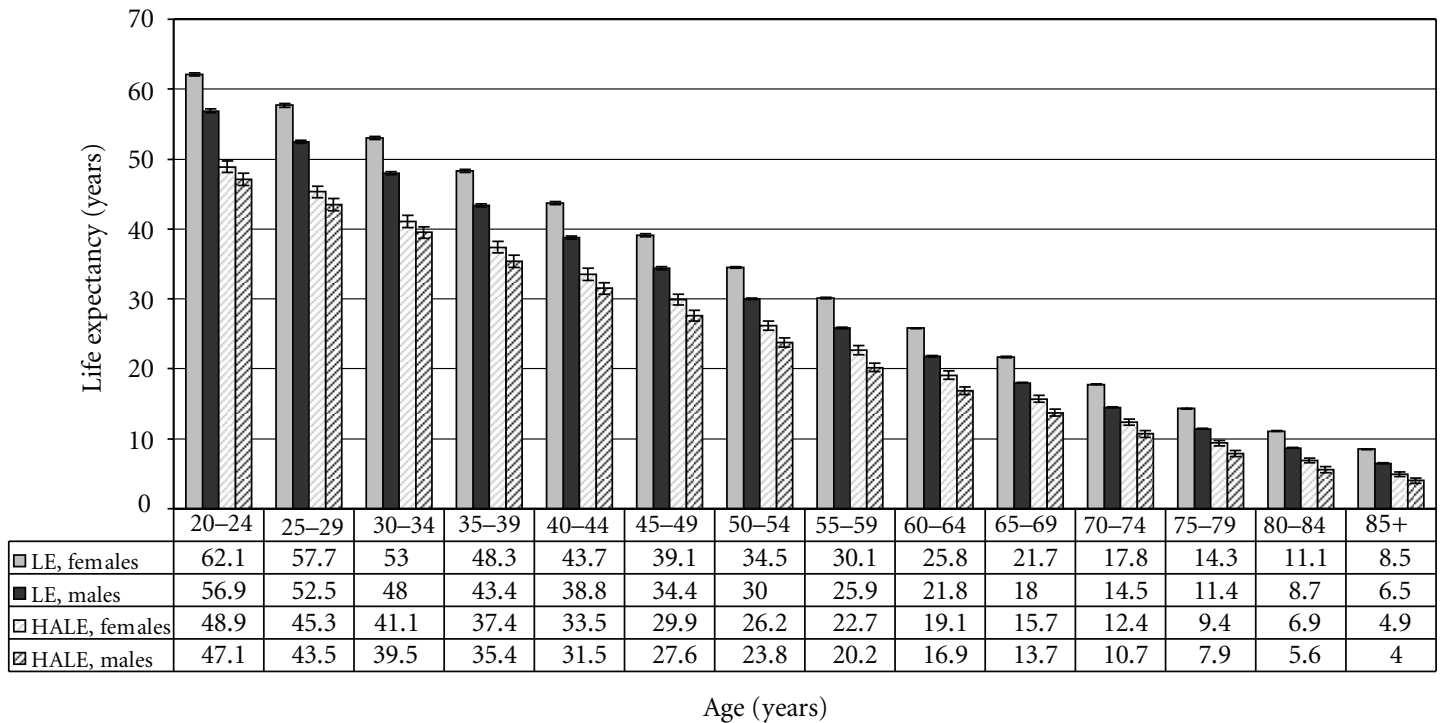

FIGURE 2: Life expectancy (LE) and health adjusted life expectancy (HALE) for females and males, with hypertension, Canada, 2004 to 2006. (Data source: Canadian community health survey data files (CCHS) from Statistics Canada, 2000-2005 and Canadian Chronic Disease Surveillance System Data Files (CCDSS) from Public Health Agency of Canada, 2004-2006. Data Files excluded Quebec, Nunavut and Northwest Territories.)

without hypertension was in a range 10.4-3.4 years for females and 8.2-2.5 years for males. When people without hypertension were compared to those with hypertension the loss in both LE and HALE decreased with increasing age for both women and men. The loss in LE decreased from 3.3 years at age 20 years down to 0.1 years at age 85 for women and from 4.1 down to 0.4 years for men. The loss in HALE decreases from 6.1 years at age 20 down to 0.3 years at age 85 for women and from 5.7 down to 0.4 years for men. All absolute differences were statistically significant $(P$-value $<.0001$ ) for all age groups except $85+$ age group. (It was not possible to calculate the significance level for the $85+$ age group.)

\section{Discussion}

In this study LE and HALE were estimated for Canadian adults (20 years old and older) with and without diagnosed hypertension by sex and fourteen 5-year age intervals. Our results show that life expectancy decreases with increasing age and that the decline is faster for LE than HALE (Figures 1 and 2). To explain this observation the age gradient in HUI 


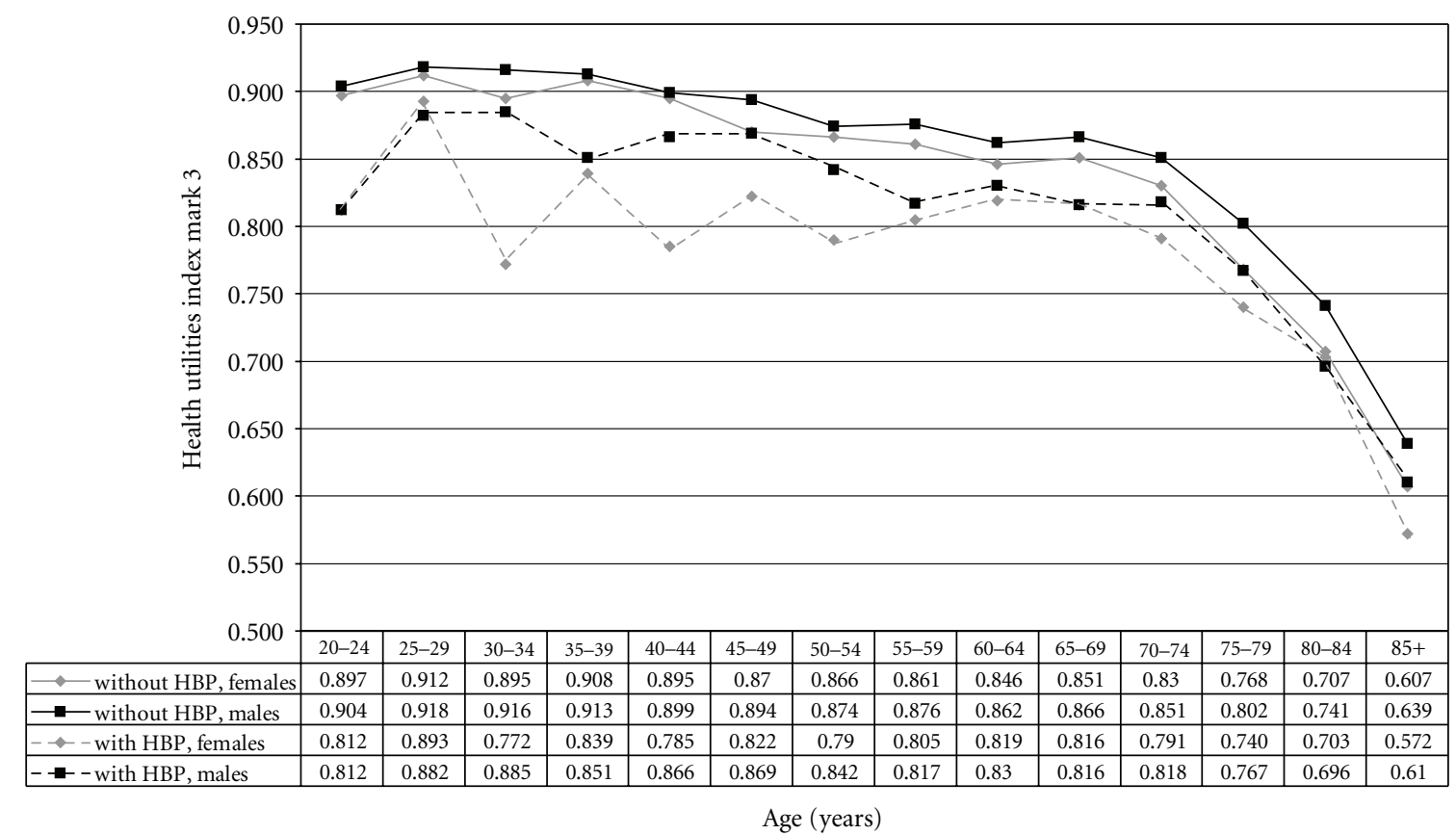

Figure 3: Health utilities index (HUI) for females and males with and without hypertension (HTN), Canada, 2000 to 2005. (Data source: Canadian Community Health Survey Data Files (CCHS) from Statistics Canada, 2000-2005. Dataset for this study excluded Quebec, Nunavut and Northwest Territories.)

scores and mortality rates was compared, which revealed, as age increased, mortality rates increased faster than the HUI scores declined. Our results also confirm that, in Canada, the LE for females is higher than for males, a direct result of higher mortality among men. It is also true for the population of people with hypertension (Table 1).

It was observed that a greater number of years of life were lost in HALE than in LE especially at younger ages (Table 2). Hypertension impacts on health and body functions to a greater extent than it impacts on mortality among young people. Differences in LE and HALE for people who was aged 85 years or older were very small and similar for both men and women. Presence or absence of hypertension at this advanced age only slightly impacted longevity and the estimated loss in life was very small.

The results attribute significant loss of health expectancy to hypertension. In reality, hypertension is a convenient marker which functions as a surrogate for health risks not controlled for in the analysis. Future work should target controlling for risk factors concurrent with a diagnosis of hypertension. That kind of study could quantify the potential impact of prevention of obesity, smoking, physical inactivity, and other risk factors associated with population health. More detailed data would be required. For instance mortality rates by BMI class would be required to evaluate the effect obesity on population health. Work plans have been prepared to explore the feasibility of controlling for obesity in HALE estimation.

The absolute difference between LE and HALE represents the number of years a person spends in poor health. Relative differences between LE and HALE quantify the portion of life a person spends in poor health. That is, the ratio of unhealthy years to the life expectancy ((LE-HALE)/LE). These measures are only meaningful within the same population (i.e., for the population of people of the same age with or without hypertension). Both measures were greater for populations of people with hypertension than for people without the disease (Table 2). Women spent more years and a greater portion of life in poor health than did men. It was observed that the number of unhealthy years decreased and the portion of life a person spends in poor health increased with increasing age. This was true for both sexes and for both diseased and not diseased populations. For example, at age 20 years, women with hypertension spent $21 \%$ of their remaining lives in poor health and that percentage gradually increased to $42 \%$ by age 85 . This age gradient in relative differences varied from $16 \%$ up to $40 \%$ for women without hypertension. The same pattern was observed for men as well but the proportions were smaller. Women with hypertension lived shorter lives and spend an even greater portion of their lives in poor health than did women without hypertension.

Differences in LE associated with hypertension were estimated for people in eastern Finland using a baseline age of 25 years by Kiiskinen et al. [4]. The loss in LE of 2.7 years for men and 2.2 years for women were reported. These estimates were lower than our estimates (3.7 men, 2.8 women, derived from Figures 1 and 2), but consistent in that the loss in life was larger for males than for females. Direct comparability with our results was hampered by differences in methodology, data used, reporting year, and characteristics of the populations studied. Our literature search did not identify any publication quantifying differences in HALE associated with hypertension. 
TABle 2: Life expectancy (LE), health-adjusted life expectancy (HALE) (With 95\% Confidence Intervals), and Loss of LE and HALE at selected ages, by hypertension (HTN) status and sex, Canada, 2004 to 2006. (Dataset for this study excluded Quebec, Nunavut and Northwest Territories.)

\begin{tabular}{|c|c|c|c|c|}
\hline Sex & Life expectancy measure & Without HTN (I) & With HTN (II) & $\begin{array}{l}\text { Loss of life expectancy associated } \\
\text { with HTN (I-II) }\end{array}$ \\
\hline \multicolumn{5}{|c|}{ At age 20 years } \\
\hline \multirow{4}{*}{ Females } & $\mathrm{LE}$ & $65.4(65.3-65.4)$ & $62.1(61.9-62.4)$ & $3.3^{*}$ \\
\hline & HALE & $55.0(54.6-55.3)$ & $48.9(48.1-49.7)$ & $6.1^{*}$ \\
\hline & LE-HALE & $10.4^{*}$ & $13.2^{*}$ & \\
\hline & (LE-HALE)/LE & 0.16 & 0.21 & \\
\hline \multirow{4}{*}{ Males } & LE & $61.0(61.0-61.0)$ & $56.9(56.7-57.2)$ & $4.1^{*}$ \\
\hline & HALE & $52.8(52.5-53.1)$ & $47.1(46.3-47.8)$ & $5.7^{*}$ \\
\hline & LE-HALE & $8.2^{*}$ & $9.8^{*}$ & \\
\hline & $($ LE-HALE)/LE & 0.13 & 0.17 & \\
\hline \multicolumn{5}{|c|}{ At age 55 years } \\
\hline \multirow{4}{*}{ Females } & $\mathrm{LE}$ & $31.6(31.6-31.7)$ & $30.1(30.1-30.2)$ & $1.5^{*}$ \\
\hline & HALE & $24.7(24.4-25.1)$ & $22.7(22.3-23.0)$ & $2.0^{*}$ \\
\hline & LE-HALE & $6.9^{*}$ & $7.4^{*}$ & \\
\hline & $($ LE-HALE)/LE & 0.22 & 0.25 & \\
\hline \multirow{4}{*}{ Males } & $\mathrm{LE}$ & $28.0(28.0-28.1)$ & $25.9(25.8-25.9)$ & $2.1^{*}$ \\
\hline & HALE & $22.9(22.6-23.2)$ & $20.2(19.8-20.6)$ & $2.7^{*}$ \\
\hline & LE-HALE & $5.1^{*}$ & $5.7^{*}$ & \\
\hline & (LE-HALE)/LE & 0.18 & 0.22 & \\
\hline \multicolumn{5}{|c|}{ At age 85 years } \\
\hline \multirow{4}{*}{ Females } & $\mathrm{LE}$ & 8.6 & 8.5 & 0.1 \\
\hline & HALE & 5.2 & 4.9 & 0.3 \\
\hline & LE-HALE & 3.4 & 3.6 & \\
\hline & (LE-HALE)/LE & 0.40 & 0.42 & \\
\hline \multirow{4}{*}{ Males } & LE & 6.9 & 6.5 & 0.4 \\
\hline & HALE & 4.4 & 4.0 & 0.4 \\
\hline & LE-HALE & 2.5 & 2.5 & \\
\hline & (LE-HALE)/LE & 0.36 & 0.38 & \\
\hline
\end{tabular}

$*$ Statistically significant at .05 .

A limitation of our study was that data for residents of long-term care facilities were not available for calculation of our estimates of life expectancy and health-adjusted life expectancy. As a result, the true values for the entire population would be somewhat lower than what we reported. It is also important to note that misclassification of hypertension status is present in both the survey data and the surveillance system data we used. In the Canadian Community Health Survey, misclassification can be due to self-reporting bias, a tendency to underreport the true disease status. In the Canadian Chronic Disease Surveillance System, misclassification can be present in geographic areas where complete data are not available. Areas with a larger proportion of salaried physicians provide the least complete data, which results in identifying fewer individuals with disease. Consequently, the disease status concordance between the two data sources varies by province and territory [20-22]. Future linkage of these two data sources (CCDSS and CCHS) would reduce self-reporting bias and misclassification error. The estimates of LE and HALE presented in this paper are based on mortality and morbidity experience of people with and without hypertension for the period of 2004-2006. They should be treated as descriptive cross-sectional statistics based on past experience rather than predictive, as the mortality and morbidity experience will change with time.

This paper described the method used by the Public Health Agency of Canada to calculate life expectancy and health-adjusted life expectancy among Canadian adults with and without hypertension, based on mortality data for 2004 to 2006 and morbidity data for 2000 to 2005. Our work shows that it is possible to calculate health-adjusted life expectancy for all Canadians and for subpopulations 
with this particular chronic condition. Our method can be adapted for calculations for other chronic conditions and diseases to provide useful information for public health researchers and policy makers.

\section{References}

[1] F. H. H. Leenen, J. Dumais, N. H. McInnis et al., "Results of the Ontario survey on the prevalence and control of hypertension," Canadian Medical Association Journal, vol. 178, no. 11, pp. 1441-1449, 2008.

[2] "Public Health Agency of Canada. Report from the Canadian Chronic Disease Surveillance System: Hypertension in Canada," 2010, http://www.phac-aspc.gc.ca/cd-mc/cvd-mcv/ ccdss-snsmc-2010/index-eng.php.

[3] K. Wilkins, N. R. Campbell, M. R. Joffres et al., "Blood pressure in Canadian adults," Health Reports, vol. 21, no. 1, pp. 37-46, 2010.

[4] U. Kiiskinen, E. Vartiainen, P. Puska, and A. Aromaa, "Longterm cost and life-expectancy consequences of hypertension," Journal of Hypertension, vol. 16, no. 8, pp. 1103-1112, 1998.

[5] O. H. Franco, A. Peeters, L. Bonneux, and C. De Laet, "Blood pressure in adulthood and life expectancy with cardiovascular disease in men and women: life course analysis," Hypertension, vol. 46, no. 2, pp. 280-286, 2005.

[6] Canadian Chronic Disease Surveillance System (CCDSS) (formerly known as National Diabetes Surveillance System, NDSS), http://www.phac-aspc.gc.ca/ccdpc-cpcmc/ndsssnsd/english/ndss_description/index-eng.php.

[7] Statistics Canada, Canadian Community Health Survey (CCHS): Detailed information for 2000-2001 (cycle 1.1), http://www.statcan.gc.ca/cgi-bin/imdb/p2SV.pl?Function=get Survey\&SurvId $=3226 \&$ SurvVer $=0 \&$ InstaId $=15282 \&$ InstaVer $=1 \&$ SDDS=3226\&lang=en $\& \mathrm{db}=\mathrm{IMDB} \& \mathrm{adm}=8 \& \mathrm{dis}=2$.

[8] Statistics Canada, Canadian Community Health Survey (CCHS): Detailed information for 2003 (cycle 2.1), http:// www.statcan.gc.ca/cgi-bin/imdb/p2SV.pl?Function=getSurvey\&SurvId $=3226 \&$ SurvVer $=0 \&$ InstaId $=15282 \&$ InstaVer $=2 \&$ SDDS=3226\&lang $=e n \& d b=I M D B \& a d m=8 \& d i s=2$.

[9] Statistics Canada. Canadian Community Health Survey (CCHS): Detailed information for 2005 (cycle 3.1), http:// www.statcan.gc.ca/cgi-bin/imdb/p2SV.pl?Function=getSurvey\&SurvId $=3226 \&$ SurvVer $=0$ \&InstaId $=15282 \&$ InstaVer $=3 \&$ SDDS=3226\&lang $=$ en $\& d b=I M D B \& a d m=8 \& d i s=2$.

[10] W. Furlong, D. Feeny, G. Torrance, and R. Barr, The Health Utilities Index (HUI3) System for Assessing Health-Related Quality of Life in Clinical Studies, Health Economic and Policy Analyses Research Paper, McMaster University Center for Health Economics and Policy Analysis, Hamilton, ON, Canada, 2001.

[11] J. Horsman, W. Furlong, D. Feeny, and G. Torrance, "The Health Utilities Index (HUI): Concepts, measurement properties and applications," Health and Quality of Life Outcomes, vol. 1, article 54, 2003.

[12] D. Yeo, H. Mantel, and T. P. Liu, "Boostrap variance estimation for the National Population Health Survey," in Proceedings of the Survey Research Methods Section, American Statistical Association, 1999.

[13] Statistics Canada, Bootvar software [bootstrapping program], http://www.statcan.ca/english/rdc/bootvar_sas.htm .

[14] S. Thomas and B. Wannell, "Combining cycles of the Canadian community health survey," Health Reports, vol. 20, no. 1, pp. 53-58, 2009.
[15] C. L. Chiang, The Life Table and Its Applications, Robert E. Krieger Company, 1984.

[16] J. J. Hsieh, "A general theory of life table construction and a precise abridged life table method," Biometrical Journal, vol. 33, no. 2, pp. 143-162, 1991.

[17] D. F. Sullivan, "A single index of mortality and morbidity," HSMHA Health Reports, vol. 86, no. 4, pp. 347-354, 1971.

[18] C. Bebbington, "Expectation of life without disability measured from the OPCS disability surveys," in Proceedings of the Health Expectancy on the 1st Workshop of the International Health Life Expectancy Network (REVES '92), J. M. Robine, M. Blanchet, and J. Dowd, Eds., pp. 23-34, MNSO, London, UK, 1992.

[19] C. Mathers, Health expectancies in Australia, 1981 and 1998, Australian Institute of Health Publications, Canberra, ACT, Australia, 1991,.

[20] K. Tu, N. R. Campbell, Z. L. Chen, K. J. Cauch-Dudek, and F. A. McAlister, "Accuracy of administrative databases in identifying patients with hypertension," Open Medicine, vol. 1, no. 1, pp. 18-26, 2007.

[21] K. Atwood, P. McCrea, and K. Reimer, "National Diabetes and Other Chronic Disease Surveillance System validation study: intersecting self-report CCHS data with NDCSS prevalence data for hypertensive cases," Report prepared for the National Diabetes and Other Chronic Disease Surveillance System, 2009.

[22] K. Atwood, P. McCrea, and K. Reimer, "National Diabetes and Other Chronic Disease Surveillance System validation study: intersecting self-report CCHS data with prevalence NDCSS data," National Diabetes and Other Chronic Disease Surveillance System, 2008. 


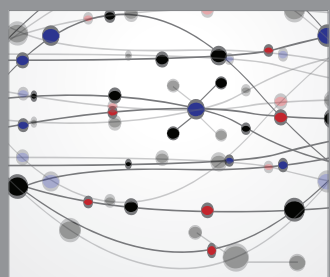

The Scientific World Journal
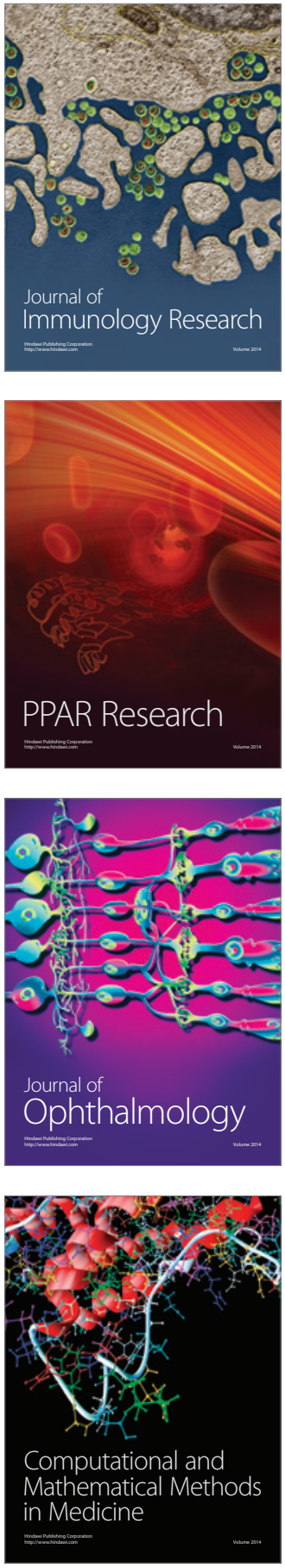

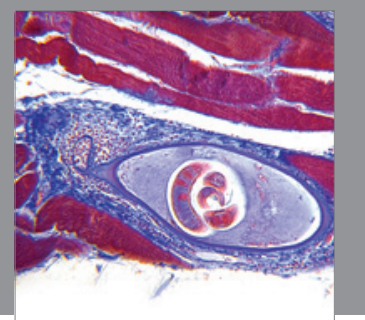

Gastroenterology

Research and Practice
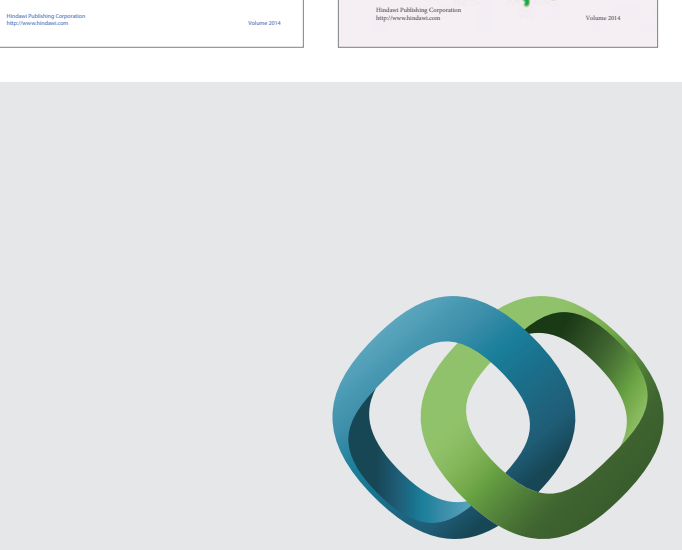

\section{Hindawi}

Submit your manuscripts at

http://www.hindawi.com


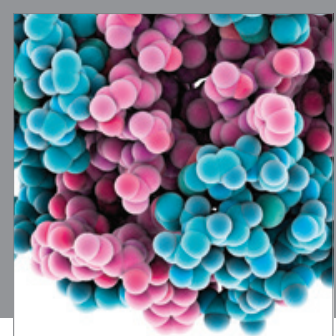

Journal of
Diabetes Research

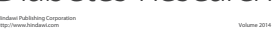

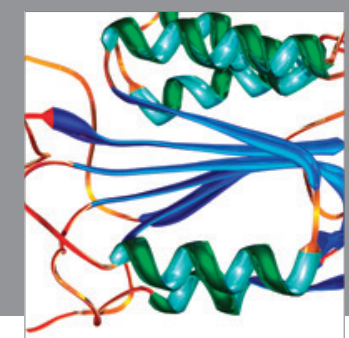

Disease Markers
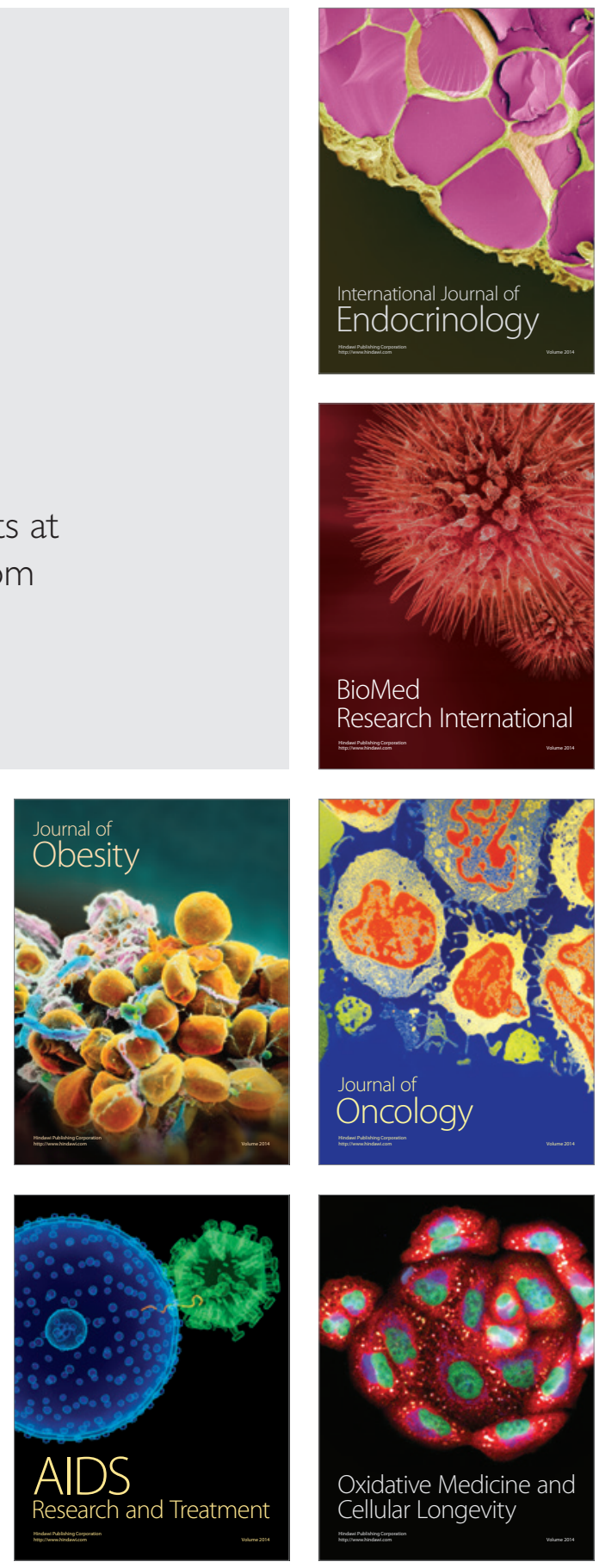